\title{
Urinary Catheters: What Type Do Men and Their Nurses Prefer?
}

\author{
Sanjay Saint, MD, MPH, "S Benjamin A. Lipsky, MD, t‡ Paul D. Baker, ARNP," \\ Linda L. McDonald, RN, MSPH, ${ }^{\ddagger}$ and Kathleen Ossenkop, ARNP, $M N^{\ddagger}$
}

OBJECTIVES: Urinary catheters are used frequently, but the relative risks and benefits of different types of devices are not clear. We sought to determine the beliefs of both older male patients and nursing staff about the relative merits and problems of condom and indwelling catheters.

DESIGN: Patient and nurse survey using convenience sampling.

SETTING: A University-affiliated Veterans Affairs medical center.

PARTICIPANTS: Men hospitalized on medical, rehabilitation and nursing home units using either an indwelling or a condom catheter were invited to participate as were all members of the nursing staff on these units. Of 116 eligible patients, 104 were interviewed (response rate $=90 \%$ ). Of 107 eligible nursing staff members, 99 completed the questionnaires (response rate $=92 \%$ ).

INTERVENTION AND MEASUREMENTS: Consenting patients were interviewed personally about their urinary catheter. The nursing staff were asked to complete a selfadministered questionnaire.

RESULTS: Patients were mostly older and predominantly hospitalized on the medical service. Compared with those using an indwelling catheter, patients using a condom catheter were more likely to believe that their catheter was comfortable ( 86 vs $58 \%, P=.04$ ) and less likely to believe it was painful ( 14 vs $48 \%, P=.008$ ) or to restrict their activity (24 vs $61 \%, P=.002)$. The nursing staff had a mean of 13 years nursing experience, and the majority worked in the nursing home unit. Most of the nursing staff respondents believed that condom catheters were less painful and restrictive for patients and were easier to apply, but they also believed that they fell off and leaked more often and required more nursing time.

CONCLUSIONS: Both patients and nursing staff prefer condom to indwelling catheters for patient comfort, but they recognize that dislodgment and leaking are major drawbacks

From the "Department of Medicine, University of Michigan Health System; †Department of Medicinc, University of Washington School of Medicinc; $¥$ Veterans Affairs Puget Sound Health Care System; and SHealth Services Research and Development, Ann Arbor Veterans Affairs Medical Center.

This work was supported, in part, by the Department of Veterans Affairs and the Robert Wood Johnson Clinical Scholars Program.

Address correspondence and reprint requests to Sanjay Saint, MD, MPH, University of Michigan Health System, 3116 Taubman Center, Box 0376, 1500 East Medical Center Drive, Ann Arbor, MI 48109-0376. E-mail: saint@umich.edu of condom catheters. A more secure condom catheter would greatly improve the management of male incontinence. J Am Geriatr Soc 47:1453-1457, 1999.

Key'words: urinary catheter; condom catheter; incontinence; comfort; urinary tract infection

$\mathrm{E}$ ach year, millions of Americans in acute care hospitals, extended care facilities, and at home use urinary collection devices. ${ }^{1}$ Although often a medical necessity, urinary catheters are a major cause of morbidity. ${ }^{2}$ In men, both indwelling and condom urinary catheters are used. Each is associated with an increased risk of urinary tract infection, ${ }^{3-8}$ but the relative risk of one versus the other is unclear. The risk of infection is a major factor when deciding which type of catheter to use for patients requiring urinary collection. Other issues, such as local genitourinary complications, catheter-associated discomfort, and urinary leakage, are also important. Keeping these issues in mind, the preference of the patient should also be considered. The views of nursing staff are also important since these health care providers often help select the type of catheter used and are usually responsible for its maintenance in both acute and chronic care settings.

Little research has been done regarding patients' attitudes about and preferences for urinary collection devices, in general, and urinary catheters, in particular. ${ }^{9-11}$ Patients must often use these catheters for prolonged periods, and they can be associated with substantial discomfort. A recent survey of three Veterans Affairs (VA) medical centers found that urinary incontinence affected $5 \%$ of acute care and $30 \%$ of long-term care patients. ${ }^{12}$ Indwelling and condom catheters were each used for $35 \%$ of these patients. ${ }^{12}$ Therefore, we sought to assess formally patient and nurse attitudes about the two types of urinary collection devices used most commonly for men in our VA medical center: indwelling urethral catheters and external (or condom) catheters.

\section{METHODS}

\section{Subjects}

To survey the attitudes of patient and nursing staff regarding urinary collection devices we developed a questionnaire for each group. Male patients housed on the medical, rehabilitation, and nursing home units of the Puget Sound VA Health Care System between May and November 1998 who were using either an indwelling or condom urinary catheter were potential candidates for the patient survey. Only those 
with a urinary catheter in use for at least 24 hours were eligible to participate in the study. A total of 146 consecutive patients were invited to participate.

All members of the nursing staff (i.e., registered nurses, licensed practical nurses, nursing assistants) on the same three units (medical, rehabilitation, and nursing home) at the VA Puget Sound Health Care System were potential candidates for the nurse survey. A total of 107 members of the nursing staff were approached by the study nurse $(\mathrm{KO})$ and invited to participate.

\section{Patient Survey}

For the patient preference component, the study nurse (KO) conducted a face-to-face interview with each patient. During pretesting, we found that patients had difficulty answering questions using a 5-point Likert scale (which ranged from strongly agree to strongly disagree). Thus, we developed a simple instrument requiring only "yes" or "no" answers for each question, which they seemed to understand adequately. Patients were asked to answer each of the five questions listed in Table 1. Patients were also asked if they remembered having another type of urinary collection device in the past (i.e., the type of urinary catheter that the patient was not currently using or a disposable diaper). For participants answering affirmatively, we asked whether they preferred the current device or the previous one.

\section{Nursing Survey}

Nursing staff members were asked to complete a twopart self-administered questionnaire. In the first part, they were asked to rate the statements in Table 2 using a 5-point Likert scale ( $1=$ strongly disagree; $3=$ neutral; $5=$ strongly agree). In the second part, nursing staff were asked to compare indwelling and condom catheters by addressing: (1) the amount of time spent managing each type of catheter during each shift; (2) the type of catheter that would be preferable if the only consideration were nursing convenience; $(3)$ and the type of catheter that would be preferable if the only consideration were patient comfort. We also obtained information about each respondent's professional licensure (i.e., nursing assistant, licensed practical nurse, registered nurse), highest level of education, years of nursing experience, and gender. Finally, we asked each respondent to estimate the number of patients with an indwelling catheter they had cared for in the past year and the number of patients with a condom catheter.

We ensured that responses to the questions were confdential for both patient and nursing staff participants. The
Institutional Review Board of the University of Washington approved the study protocol. Copies of the complete surveys are available from the authors on request.

\section{Statistical Analysis}

Chi-square was used for comparing categorical variables, Student's $t$ test was used for comparing continuous variables, and multivariable logistic regression was used to model respondents' preferences for one type of urinary collection versus another. All analyses were performed using SPSS for the IBM (SPSS version 8.0; Chicago, IL).

\section{RESULTS}

\section{Patient Sample}

Among 146 patients who were potential candidates for this study, 30 were deemed ineligible because they had delirium or dementia severe enough to render them unable to comprehend the questions. Of the 116 eligible patients, 104 completed the interviews (response rate $90 \%$ ). Participants were mostly older men (mean age $=71$ years; standard deviation $(S D)=12$ ). Seventy-two percent of participants were hospitalized on an acute care medical ward, and the rest were on other services (i.e., nursing home, surgery, neurology, rehabilitation). Approximately $80 \%$ were using an indwelling urethral catheter.

\section{Nursing Sample}

Of the 107 eligible nursing staff members, 99 participated in the survey (response rate $92 \%$ ). Almost $80 \%$ were women, and they averaged 13 years of nursing experience $(\mathrm{SD}=12)$. There was an approximately equal division of nursing licenses among nursing assistants, licensed practical nurses, and registered nurses (roughly $30 \%$ each); $8 \%$ had advanced training as a nurse practitioner or had received a master's degree. Almost two-thirds of the respondents worked in the nursing home, with about one-fifth each on the rehabilitation unit and the medical ward. Almost threequarters had taken care of at least 10 patients with a condom catheter, and more than half had cared for at least 10 patients with an indwelling catheter in the past year.

\section{Urinary Catheter Preference - Patients}

The average age for patients using indwelling catheters (70.8 years) was similar to those using condom catheters (70.9 years). In addition, the proportion of men using indwelling catheters did not differ significantly by hospital services $(P=.72)$.

Table 1. Results of the Patient Interviews

Proportion of Condom

Catheter Users

Answering "Yes"

Question

\section{Proportion of Indwelling \\ Catheter Users \\ Answering "Yes"}

$(\mathrm{N}=83)$
$P$ Value

Is the current urinary catheter. . . comfortable?

painful?

convenient?

$86 \%$

$14 \%$

$86 \%$

$24 \%$

restricting your daily activity?

$24 \%$
$58 \%$

$48 \%$

$75 \%$

$61 \%$

$30 \%$
.04

.008

.40

.002

.50 
Table 2. Results of Questionnaires Administered to Nursing Staff

\begin{tabular}{lccc}
\hline \multicolumn{1}{c}{ Statement } & $\begin{array}{c}\text { Agree or } \\
\text { Strongly Agree } \\
\mathrm{N}(\%)\end{array}$ & $\begin{array}{c}\text { Neutral } \\
\mathrm{N}(\%)\end{array}$ & $\begin{array}{c}\text { Disagree or } \\
\text { Strongly Disagree } \\
\mathrm{N}(\%)\end{array}$ \\
\hline $\begin{array}{l}\text { Compared with indwelling catheters. . } \\
\text { condom catheters are less comfortable for patients }\end{array}$ & $23(23 \%)$ & $31(31 \%)$ & $44(44 \%)$ \\
condom catheters are more painful for patients & $14(14 \%)$ & $20(20 \%)$ & $64(65 \%)$ \\
condom catheters are more convenient for patients & $44(44 \%)$ & $26(26 \%)$ & $28(28 \%)$ \\
condom catheters are more restrictive of patients daily activity & $13(13 \%)$ & $32(32 \%)$ & $53(54 \%)$ \\
condom catheters are more embarrassing for patients & $13(13 \%)$ & $43(43 \%)$ & $42(42 \%)$ \\
condom catheters are easier to put on & $57(58 \%)$ & $16(16 \%)$ & $25(25 \%)$ \\
condom catheters fall off more often & $95(96 \%)$ & $2(2 \%)$ & $1(1 \%)$ \\
condom catheters leak less often & $7(7 \%)$ & $11(11 \%)$ & $80(81 \%)$ \\
patients who use condom catheters need their sheets & $73(74 \%)$ & $13(13 \%)$ & $12(12 \%)$ \\
$\quad$ changed more often & $7(7 \%)$ & $16(16 \%)$ & $75(76 \%)$ \\
patients who use condom catheters need their clothes & & & \\
$\quad$ changed less often & & & \\
\hline
\end{tabular}

Table 1 shows the main results of the patient interviews. Compared with users of indwelling catheters, condom catheter users were significantly more likely to believe that the condom catheter was more comfortable, less painful, and less restricting on activities of daily living. There were no significant differences between condom and indwelling catheter users regarding questions about catheter convenience or associated embarrassment.

We performed logistic regression analysis using each dichotomous answer (yes or no) as the dependent variable with patient age, hospital service, and current catheter type (indwelling or condom) as independent variables. Compared with indwelling catheters, condom catheters were found to be more comfortable (odds ratio (OR) $=4.2 ; 95 \%$ confidence interval $(\mathrm{CI}), 1.1-15.6 ; P=.03$ ), less painful (OR $=0.17$; $95 \% \mathrm{CI}, 0.05$ to $0.64 ; P=.008$ ), and less restrictive (OR $0.23 ; 95 \% \mathrm{CI}, 0.07-0.75 ; P=.01)$. Catheter type was not found to be significantly related to either convenience or embarrassment.

Thirty-six patients had personal experience with the type of urinary catheter (indwelling or condom) that they were not currently using. Of this subgroup, $17(47 \%)$ preferred a condom catheter, $14(39 \%)$ preferred an indwelling catheter, and five $(14 \%)$ did not state a preference. Of the 14 patients using a condom catheter at the time of the interview, all indicated that they preferred this to an indwelling catheter. Of the 22 patients currently using an indwelling catheter, 14 preferred an indwelling catheter, three preferred a condom catheter, and five had no preference.

Another method of managing urinary incontinence, disposable diapers, had been used by 27 patients. Of these, all 10 of the patients that were currently using a condom catheter stated that they preferred this device. Of the 17 that were using an indwelling catheter at the time of the interview, nine $(53 \%)$ preferred an indwelling catheter, four $(24 \%)$ preferred diapers, and four (24\%) had no preference.

Of the 43 patients who had personal experience with condom catheters (either currently using them or had in the past), seven (16\%) offered spontaneously that the main drawback with condom catheters was the associated leaking.

\section{Urinary Catheter Preference - Nursing Staff}

Table 2 shows the main results of the nursing staff questionnaire. The responses to each statement were divided into three categories for ease of presentation. "Agree" and "strongly agree" were grouped into one category, "disagree" and "strongly disagree" into a second, and "neutral" into a third. The nursing staff believed that from the patient's perspective, condom catheters are more comfortable, less painful, less restrictive, less embarrassing, and somewhat more convenient than indwelling catheters. Compared with indwelling catheters, the nursing staff believed that condom catheters are easier to put on, but they fall off more often, leak more often, and require patients to have their clothes and sheets changed more often.

The nursing staff reported that they spent approximately 5 to 10 more minutes per shift managing the condom catheter than the indwelling catheter $(P=.003)$.

When asked what would be their choice of catheter if the only consideration were nursing convenience, $41(41 \%)$ of all respondents preferred the indwelling catheter, $40(40 \%)$ preferred the condom catheter, and $15(15 \%)$ stated that it did not matter. If patient comfort were the only consideration when deciding between the two types of devices, $54(54 \%)$ respondents preferred the condom catheter, $26(26 \%)$ preferred the indwelling catheter, and $16(16 \%)$ had no preference.

We also performed multivariable logistic regression analyses to delineate predictors for a specific catheter preference, after removing those respondents who did not state a preference. Our models included the respondent's type of licensure, nursing experience in years, gender, hospital unit, time spent managing each type of catheter, and experience with each type of catheter in the past year as possible independent predictors; the dependent variable was condom catheter (coded as " 0 ") or indwelling catheter (coded as " 1 "). When considering nursing convenience, the only significant predictor was the number of patients with condom catheters that the nurse had cared for in the past year (OR $=0.54 ; 95 \%$ $\mathrm{CI}, 0.30$ to $0.98 ; P=.04)$; the greater the experience with condom catheters, the more likely the nurse would prefer 
them. When considering patient comfort, the important predictors were the amount of time spent on each type of urinary catheter per shift. As the number of minutes spent managing the indwelling catheter increased, the more likely the respondent was to prefer the condom catheter $(\mathrm{OR}=0.34 ; 95 \% \mathrm{CI}$, $0.12-0.97 ; P=.04)$. On the other hand, as the number of minutes spent managing the condom catheter increased, the more likely the respondent was to prefer the indwelling catheter (OR $=2.06 ; 95 \% \mathrm{Cl}, 0.95-4.44 ; P=.07)$.

\section{DISCUSSION}

Indwelling and condom catheters are often used to collect urine in men with urinary incontinence. ${ }^{13-15}$ The relative merits of and problems with these catheters have not been well studied. In particular, little is known about what patients who use these devices think about them. Similarly, the views of the nursing staff, who are primarily responsible for inserting and maintaining urinary catheters, are not well known. Using parallel surveys, we attempted to gain insight into the beliefs of each of these groups.

Our patient survey indicates that men find the condom catheter more comfortable, less painful, and less restrictive on their activities. The major negative aspect of these devices is urinary leaking. The nursing staff also prefers condom catheters for patient comfort, but they believe that urinary leaking is the major problem with these devices.

It is striking that almost half of patients with indwelling catheters found these catheters painful, and almost twothirds believed that these catheters restrict their daily activity. The importance of these findings is magnified by the extent of indwelling catheter use in both acute and chronic care settings. Up to $25 \%$ of hospitalized patients receive an indwelling catheter at some point during their stay, ${ }^{16}$ and approximately 1 in 15 nursing home residents have an indwelling catheter at any given time. ${ }^{13,14}$ Considering the discomfort caused by indwelling catheters, it is of concern that up to half of catheterized patient-days in acute care settings are inappropriate. ${ }^{17,18}$

Spontaneous patient comments during the interviews provide an illustrative supplement to the structured questionnaires. Several patients offered that the indwelling catheter was painful, with two men independently stating that it "hurts like hell." The main drawback to condom catheters remarked upon by our interviewees was that they leaked. Some expressed discontent with both types of catheters; however, many recognized that they are "a means to an end," or "a necessary evil." Some even liked the reassurance and comfort the catheter provided in dealing with their urinary incontinence.

The few studies comparing condom and indwelling catheters have largely focused on the important outcome of catheter-related infection. The available data suggest that condom catheters may be associated with a lower risk of bacteriuria than indwelling catheters. ${ }^{19}$ One prospective study conducted in a VA medical center ${ }^{20}$ found that the risk of developing bacteriuria was approximately $12 \%$ per month in men wearing a condom catheter. The rate was substantially higher, however, in those who frequently manipulated their catheter. ${ }^{20}$ In two parallel cohort studies in a VA nursing home, the incidence of symptomatic urinary tract infection was about 2.5 times greater in men with a chronic indwelling catheter than in those wearing a condom catheter. ${ }^{6,7}$ On the other hand, a recent cross-sectional study in
Denmark reported that the risk of urinary tract infection in hospitalized patients was higher in those wearing condom catheters than in patients using indwelling catheters. ${ }^{3}$

These conflicting data on infection risk indicate that other factors, including cost and patient preference, should also be taken into consideration. A recent survey of three VA medical centers estimated that the total cost related to the management of urinary incontinence was between $\$ 129$ and $\$ 157$ per day. ${ }^{12}$ Urinary catheter-related costs were 100 -fold higher for condom catheter users than for those with indwelling catheters, almost entirely related to nursing time required for catheter care. ${ }^{12}$ In addition to the relative infectious risks and costs of these devices, it is crucial that patient preference for the type of catheter be taken into account; patients often use a urinary collection device for extended periods. The opinions of nurses are also important in deciding what type of catheter is most appropriate as they often select and usually maintain the device. Unfortunately, there is little data regarding patient and nurse preference. McMurdo and colleagues performed an economic analysis comparing indwelling catheterization and pads in incontinent women in which patient and nurse preference for these devices was assessed. ${ }^{11}$ These investigators found that of the 35 patients queried, 12 preferred catheters, 12 preferred pads, and 11 were undecided. The nurses in their study, however, preferred the incontinence pads. ${ }^{11}$

Our study has several limitations. First, both the patient and nurse surveys were conducted at one VA medical center. Second, we did not include women in the patient survey since external urinary collection devices have been used almost exclusively in men. Third, we did not assess patient comorbidity, and, thus, the findings from the patient survey could potentially be confounded by disease burden if the men given indwelling catheters were more ill than those given condom catheters. Fourth, our evaluation did not include informed decision-making in which the relative risks and benefits of each type of catheter are described. Given the probably greater medical morbidity associated with indwelling catheters, this type of discussion would likely lead to greater preference for condom catheters. Finally, we did not compare urinary catheters to other management strategies used in managing incontinence, such as pads, regular toileting, and intermittent catheterization.

The relative advantages and disadvantages of different urinary collection devices are unclear. Although rates of infection and other complications require further investigation, we do provide data about the important issue of patient preference. We found that patients believe that condom catheters are less painful, more comfortable, and less restrictive than indwelling catheters. Nurses also believe that condom catheters are preferable from the viewpoint of patient comfort. The main drawback with condom catheters is that they tend to leak urine. A condom catheter that is less likely to leak and to fall off would, therefore, be a welcome advance.

\section{ACKNOWLEDGMENTS}

The authors thank Dimitri A. Christakis, MD, MPH, for his thoughtful review of an earlier draft of this manuscript, and the patients and nurses who participated in the study.

\section{REFERENCES}

1. Warren JW. Catheter-associated urinary tract infections. Infect Dis Clin North Am 1997;11:609-622. 
2. Saint $S$. The clinical and economic consequences of nosocomial catheterrelated bacteriuria. Am J Infect Control, in press.

3. Zimakoff J, Stickler DJ, Pontoppidan B, Larsen SO. Bladder management and urinary tract infections in Danish hospitals, nursing homes, and home care: A national prevalence study. Infect Control Hosp Epidemiol 1996;17: 215-221.

4. Warren JW. Urethral catheters, condom catheters, and nosocomial urinary tract infections. Infect Control Hosp Epidemiol 1996;17:221-214.

5. Stamm WE. Cathcter-associated urinary tract infections: Epidemiology, pathogenesis, and prevention. Am J Med 1991;91:65-71S.

6. Ouslander JG, Greengold B, Chen S. Complications of chronic indwelling urinary catheters among male nursing home patients: A prospective study. J Urol 1987;138:1191-5.

7. Ouslander JG, Greengold B, Chen S. External catheter use and urinary tract infections among incontinent male nursing home patients. J Am Geriatr Soc 1987;35:1063-1070.

8. Kunin CM. Can we build a better urinary catheter? (editorial). N Engl J Med 1988;319:365-366.

9. Roe BH, Reid FJ, Brocklehurst JC. Comparison of four urine drainage systems. J Adv Nurs 1988;13:374-382.

10. Roe BH, Brocklehurst JC. Study of patients with indwelling catheters. J Adv Nurs 1987;12:713-18.

11. McMurdo ME, Davey PG, Elder MA et al. A cost-effectiveness study of the management of intractable urinary incontinence by urinary catheterisation or incontinence pads. J Epidemiol Community Health 1992;46:222-226.
12. Armstrong EP, Ferguson TA. Urinary incontinence: Healthcare resource consumption in veterans affairs medical centers. Veterans Health Syst J 1998; October:37-42.

13. Warren JW, Steinberg L, Hebel JR, Tenney JH. The prevalence of urethral catheterization in Maryland nursing homes. Arch Intern Med 1989;149:1535-1537.

14. Brandeis GH, Baumann MM, Hossain M et al. The prevalence of potentially remediable urinary incontinence in frail older people: A study using the Minimum Data Set. J Am Geriatr Soc 1997;45:179-184.

15. Ouslander JG, Fowler E. Management of urinary incontinence in Veterans Administration nursing homes. J Am Geriatr Soc 1985;33:33-40.

16. Haley RW, Hooton TM, Culver DH et al. Nosocomial infections in U.S. hospitals, 1975-1976: Estimated frequency by selected characteristics of patients. Am J Med 1981;70:947-959.

17. Hartstein AI, Garber SB, Ward TT et al. Nosocomial urinary tract infection: A prospective evaluation of 108 catheterized patients. Infect Control 1981;2: 380-386.

18. Jain P, Parada JP, David A, Smith LG. Overuse of the indwelling urinary tract catheter in hospitalized medical patients. Arch Intern Med 1995;155: 1425-1429.

19. Saint S, Lipsky BA. Preventing catheter-related bactcriuria: Can we? Should we? How? Arch Intern Med 1999;159:800-808.

20. Hirsh DD, Fainstein V, Musher DM. Do condom catheter collecting systems cause urinary tract infection? JAMA 1979;242:340-341. 